\title{
MRSA: new troubles from the animal farm
}
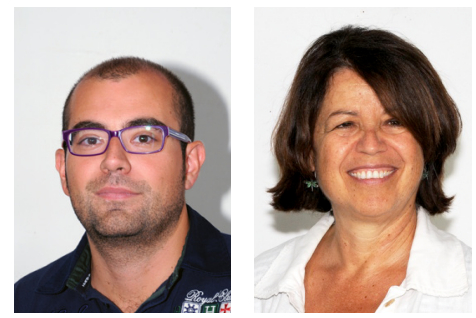

"...the pool of resistance genes

and their variants appears to be limitless ... we can expect new surprises

and new troubles and we must

keep watching."

Andrea Sanchinil \& Annalisa Pantosti ${ }^{\dagger 1}$

'Department of Infectious, Parasitic \& Immune-Mediated Diseases, Istituto Superiore di Sanità, Viale Regina Elena 299, 00161 Rome, Italy

†Author for correspondence: Tel.: +39 0649902852 = Fax: +39 0649902886 m annalisa.pantosti@iss.it

MRSA is considered the prototype of the multiple-drug resistant organisms, and represents a threat in both a hospital setting and in the community worldwide, causing serious, often difficult-to-treat infections [1]. Resistance to methicillin, and to the other $\beta$-lactam antibiotics, is caused by the presence of an additional penicillin-binding-protein (PBP), designated PBP2a. PBPs are key enzymes in the construction and maintenance of the bacterial cell wall. While the wild-type enzymes are inhibited by $\beta$-lactams, in MRSA PBP2a can exert its activity unhindered, owing to its low affinity for $\beta$-lactams, replacing the functions of the other PBPs [2]. PBP $2 \mathrm{a}$ is encoded by the mecA gene, which can be regarded as the hallmark of MRSA. mecA is embedded in a mobile genetic element, named staphylococcal cassette chromosome (SCC) mec, which often contains additional resistance genes and is organized in different structural types [3].

In the last 15 years great progress has been achieved into the understanding of the variety of genetic lineages or clones of MRSA, their diffusion in different hosts and their pathogenic potential thanks to the application of molecular typing on a large scale. It is by the powerful tool of molecular typing that the new strains causing infections in the community (designated communty-associated MRSA) have been recognized as distinct from the traditional nosocomial strains [4]. Furthermore, it is molecular typing that made it possible to rapidly pick out the emergence of 'pig' MRSA. The finding that veterinarians and pig farmers in Europe were colonized by the same clone of MRSA, characterized as sequence type (ST)398 [5], and occasionally suffered infections from this same strain, prompted the search for MRSA in farmed pigs. MRSA ST398 was found to colonize the pig nostrils and to spread and settle in the dust of the pens. As well as pigs, MRSA ST398 was found to colonize other farm animals, including veal calves and chickens, and was therefore designated livestock-associated (LA)-MRSA [6].

Now cattle is the culprit: two recent articles, one published in the Lancet Infectious Diseases and the other in Antimicrobial Agents and Chemotherapy, point at the possible transmission of MRSA strains with a novel mecA gene from bovine mastitis to humans $[7,8]$. Bovine mastitis is an important cause of economical loss in milk production and Staphylococcus aureus is one of the most common bacterial pathogens encountered [9]. Although MRSA was reported as the cause of an outbreak of mastitis in dairy cows in Belgium as far back as in 1972 [10], in several subsequent surveys the frequency of MRSA in bovine mastitis has remained low [11]. The study published in the Lancet Infectious Diseases was motivated by the results of a survey performed by the Veterinary Laboratories Agency of Weybridge, UK, which aimed at characterizing $S$. aureus strains isolated from cattle. Interestingly, out of 954 strains from bovine mastitis milk, no MRSA was detected using a mecA-based PCR assay, while 24 strains proved to be resistant to oxacillin (an analog of methicillin) by the traditional antibiotic susceptibility testing [7]. These discrepant results prompted Laura Garcia-Alvarez and collaborators to sequence one of the mecA-negative bovine MRSA, identifying a novel variant of mec $A$ that was named mec $A_{\mathrm{LGA} 251}$ after the designation of the strain. This novel mecA was in turn located in a novel SCCmec element, designated SCCmec type XI. Since mec $A_{\mathrm{LGA} 251}$ showed only $70 \%$ DNA identity with the classical mec $A$ gene, isolates carrying it could not be recognized by the current mecA-based PCR methods, including commercially available systems, nor by slide agglutination methods employing monoclonal antibodies directed to PBP2a, the protein

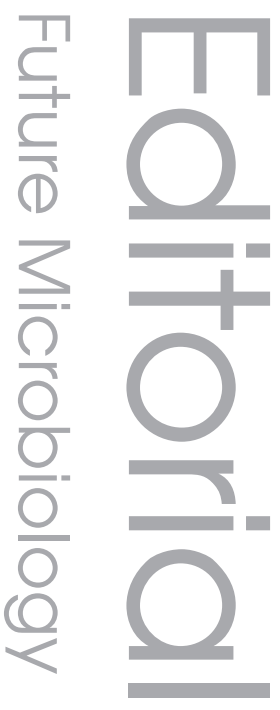

\section{Keywords}

- animals $=$ cattle $=\mathrm{CCl} 130$

- mecA $=$ MRSA $=$ SCCmecX

- ST398 $=$ Staphylococcus

aureus

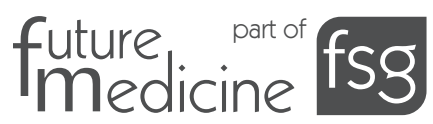


product of mecA. Following this finding, the authors started to search for isolates with the same characteristics - phenotypical MRSA that were $m e c A$ negative - in a large collection of human isolates from the UK and Denmark. They found indeed a few MRSA strains carrying $m e c A_{\mathrm{LGA} 251}$, originating from a variety of human infections, ranging from skin and soft tissue infections to sepsis.

Shore and co-authors of the study published in Antimicrobial Agents and Chemotherapy followed a different track [8]. They started with the observation of two patients admitted to different hospitals in Ireland infected with MRSA isolates that were found to be mecA-negative by PCR analysis. Sequencing of one isolate led to the identification of a novel mecA gene that was subsequently found to correspond to $m e c A_{\mathrm{LGA} 251}$. Molecular typing of the two clinical isolates by multilocus sequence typing revealed that both belonged to clonal complex (CC) 130 , a lineage only identified in animals (especially cattle) to date, suggesting a bovine origin. Indeed $\mathrm{CC} 130$ is by and large the most frequent lineage identified among the bovine and human $m e c A_{\mathrm{LGA} 251}$-positive MRSA in the Lancet Infectious Diseases study [7].

Two important messages arise from these two papers. The first is that in a few years farm animals have fuelled new MRSA strains into the human disease arena: LA-MRSA from pigs and now $m e c A_{\mathrm{LGA} 251}$-carrying MRSA from cattle. In addition, strains from animal sources have provided the reservoirs of resistance genes that have enriched the large array of MRSA: the last in order of appearance is the $c f r$ gene that confers resistance to several antibiotic classes, including the therapeutically precious oxazolidinones [12]. Originally discovered in other species of staphylococci, $c f r$ has disseminated into human-adapted MRSA [13]. It is possible that $S$. aureus from farm animals had been causing human diseases for many years, but this was impossible to ascertain before the molecular typing era. As a matter of fact, one of the first cases of human infections caused by mec $A_{\mathrm{LGA} 251}$-MRSA dates back to 1975 [7]. However, it is also possible that the style of intense farming together with the continuous pressure exerted by antibiotic use, both among animals and humans, have favored the selection and spread of resistant strains that have broken the species-specificity constraints and are able to infect different groups of living beings.

The second message has very broad implications, because the discovery of $m e c A_{\mathrm{LGA251}}$-positive MRSA is a clear example of the limitation of molecular diagnostics. PCR-based methods are sometimes advocated as the methods of choice for confirming an MRSA diagnosis and for screening patients infected with MRSA in some settings because they are fast and accurate [14]. However, the problem with molecular diagnosis is that we can only detect what is already known. At present the frequency of $m e c A_{\mathrm{LGA} 251}$-carrying MRSA in human infections is estimated to be very low, approximately $0.6 \%$ of all MRSA infections according to data from Denmark [7]. However, the risk of overlooking an MRSA infection or colonization cannot be dismissed. The central message we gather from this new finding is that a robust capability to perform antibiotic sensitivity testing must be maintained and relied upon. Antibiotic resistance is the phenotypic result of possible diverse genotypes, and clinical decisions must be based on the best possible laboratory results. We know today through extensive genome sequencing, that the pool of resistance genes and their variants appears to be limitless and the flow of genes between the environment, animals and humans is continuous. Therefore, we can expect new surprises and new troubles and we must keep watching.

\section{Financial \& competing interests disclosure \\ The authors have no relevant affiliations or financial involvement with any organization or entity with a financial interest in or financial conflict with the sub- ject matter or materials discussed in the manuscript. This includes employment, consultancies, honoraria, stock ownership or options, expert testimony, grants or patents received or pending, or royalties. \\ No writing assistance was utilized in the production of this manuscript.}

\section{Bibliography}

1. Pantosti A, Venditti M. What is MRSA? Eur. Respir. J. 34, 1190-1196 (2009).

2. Pantosti A, Sanchini A, Monaco M. Mechanisms of antibiotic resistance in Staphylococcus aureus. Future Microbiol. 2, 323-334 (2007).
3. International Working Group on the Classification of Staphylococcal Cassette Chromosome Elements (IWG-SCC). Classification of staphylococcal cassette chromosome mec (SCCmec): guidelines for reporting novel SCCmec elements. Antimicrob. Agents Chemother. 53, 4961-4967 (2009).
4. Deleo FR, Otto M, Kreiswirth BN, Chambers HF. Community-associated meticillin-resistant Staphylococcus aureus. Lancet 375, 1557-1568 (2010).

5. Wulf M, Voss A. MRSA in livestock animals - an epidemic waiting to happen? Clin. Microbiol. Infect. 14, 519-521 (2008). 
6. Cuny C, Friedrich A, Kozytska S et al. Emergence of methicillin-resistant Staphylococcus aureus (MRSA) in different animal species. Int. J. Med. Microbiol. 300, 109-117 (2010).

7. García-Álvarez L, Holden MT, Lindsay H et al. Meticillin-resistant Staphylococcus aureus with a novel mecA homologue in human and bovine populations in the UK and Denmark: a descriptive study. Lancet Infect. Dis. 11, 595-603 (2011).

8. Shore AC, Deasy EC, Slickers P et al. Detection of staphylococcal cassette chromosome mec type XI carrying highly divergent mecA, mecI, mecR1, $b l a Z$, and $c c r$ genes in human clinical isolates of clonal complex 130 methicillin- resistant Staphylococcus aureus. Antimicrob. Agents Chemother. 55, 3765-3773 (2011).

9. Bradley AJ, Leach KA, Breen JE, Green LE, Green MJ. Survey of the incidence and aetiology of mastitis on dairy farms in England and Wales. Vet. Rec. 160, 253-258 (2007).

10. Devriese LA, Hommez J. Epidemiology of methicillin-resistant Staphylococcus aureus in dairy herds. Res. Vet. Sci. 19, 23-27 (1975).

11. Huber H, Koller S, Giezendanner N, Stephan R, Zweifel C. Prevalence and characteristics of meticillin-resistant Staphylococcus aureus in humans in contact with farm animals, in livestock, and in food of animal origin, Switzerland, 2009. Euro Surveill. 15(16), 19542 (2010).
12. Long KS, Poehlsgaard J, Kehrenberg C, Schwarz S, Vester B. The Cfr rRNA methyltransferase confers resistance to phenicols, lincosamides, oxazolidinones, pleuromutilins, and streptogramin $\mathrm{A}$ antibiotics. Antimicrob. Agents Chemother. 50, 2500-2505 (2006).

13. Morales G, Picazo JJ, Baos E et al. Resistance to linezolid is mediated by the $c f r$ gene in the first report of an outbreak of linezolid-resistant Staphylococcus aureus. Clin. Infect. Dis. 15, 821-825 (2010).

14. Harbarth S, Hawkey PM, Tenover F, Stefani S, Pantosti A, Struelens MJ. Update on screening and clinical diagnosis of methicillin-resistant Staphylococcus aureus (MRSA). Int. J. Antimicrob. Agents 37, 110-117 (2011). 